\title{
Photo-movement in the sea anemone Aiptasia influenced by light quality and symbiotic association
}

\author{
Shawna A. Foo ${ }^{1,2}$ (10) Lauren Liddell $^{3} \cdot$ Arthur Grossman $^{4} \cdot$ Ken Caldeira $^{1}$
}

Received: 9 January 2019/Accepted: 10 October 2019/Published online: 13 November 2019

(C) The Author(s) 2019

\begin{abstract}
The relationship between cnidarians and their micro-algal symbionts is crucial for normal animal function and the formation of coral reefs. We used the sea anemone Exaiptasia pallida (Aiptasia) as a model cnidarian-dinoflagellate system to determine the effects of white, blue and red light on photo-movement. In white light, phototropism and phototaxis of Aiptasia were dependent on the presence of symbionts; anemones with symbionts bent and moved toward the light, whereas aposymbiotic anemones (lacking algal symbionts) moved, but without strong directionality. Phototaxis and phototropism also occurred in blue light, but to a lesser extent than in white light, with no apparent response to red light. Phototactic behavior was also sensitive to the specific anemone-symbiont pairing. The ability to sense and move in response to light would presumably allow for selection of favorable habitats. Overall, this study demonstrates that the algal symbiont is required for photo-movement of the host and that the extent
\end{abstract}

Topic Editor Simon Davy

Electronic supplementary material The online version of this article (https://doi.org/10.1007/s00338-019-01866-w) contains supplementary material, which is available to authorized users.

Shawna A. Foo

sfoo@asu.edu

1 Department of Global Ecology, Carnegie Institution for Science, Stanford, CA, USA

2 Center for Global Discovery and Conservation Science, Arizona State University, Tempe, AZ, USA 85287

3 Space Biosciences, NASA Ames Research Center, Moffett Field, CA, USA

4 Department of Plant Biology, Carnegie Institution for Science, Stanford, CA, USA of movement is influenced by the different anemonesymbiont associations.

Keywords Aiptasia - Phototaxis - Cnidarian-dinoflagellate symbiosis · Coral reefs

\section{Introduction}

The development and persistence of coral reefs depend on the relationship between scleractinian corals and their photosynthetic dinoflagellate symbionts. This symbiotic partnership is highly susceptible to destabilization where the breakdown of the symbiosis between the cnidarian host and the algae, termed "bleaching," represents a loss of algae from host tissue, an event that is a major threat to the viability of coral reefs worldwide (Anthony et al. 2007; Hoegh-Guldberg et al. 2007; Roth 2014; Warner and Suggett 2016). Bleaching of symbiotic cnidarians can occur in response to pollution, disease, the absorption of excess excitation energy and elevated temperature (Brown 1997). Symbiotic cnidarians have adapted to a wide range of photic environments, with exposure to different light regimes that vary over seasons and with depth in the water column (Gattuso et al. 1995). Furthermore, the holobiont has various photoreceptors (chromophore-associated proteins) including the blue-green light-absorbing rhodopsins, potentially the red/far red light-absorbing photoreversible phytochromes and the blue light-absorbing cryptochromes and phototropins (Duanmu et al. 2017). These photoreceptors detect light signals and elicit downstream responses associated with circadian rhythms, photomorphogenesis and other physiological responses (Somers et al. 1998; Delvin and Kay 2000; Christie et al. 2015; Kong and Okajima 2016).

Symbiotic cnidarians can show distinct behavioral changes in response to light including positive phototaxis, 
expansion and contraction of tentacles (Sawyer et al. 1994; Gorbunov and Falkowski 2002), and negative phototaxis in high sunlight (Shick 1991). Light characteristics also have a profound influence on cnidarian physiology ( $\mathrm{Su}$ et al. 2014) with differential effects of light qualities on the control of the circadian machinery and the photosynthetic efficiency of its endosymbiotic alga (Hastings and Sweeny 1960; López-Figueroa et al. 2003). For the coral species Porites lutea and Acropora variabilis, blue light enhanced calcification rates (Cohen et al. 2016). For another coral, Stylophora pistillata, exposure to red light caused a decrease in survival and symbiont densities (Wijgerde et al. 2014). Additionally, photosynthetic rhythmicity in freeliving dinoflagellates in the family Symbiodiniaceae is impacted by blue and red light through shortening and lengthening of cycle times (Yacobovitch et al. 2004; Sorek and Levy 2012), which suggests that the activities of the algae can be controlled by light quality. Furthermore, the light environment influences the distribution and structure of Symbiodiniaceae communities where corals harbor distinct algal types adapted to specific light regimes (Iglesias-Prieto et al. 2004).

Phototropism (orientation of the organism toward or away from light) and phototaxis (movement of the entire organism toward or away from light) are important lightdriven movements that may allow cnidarians to favorably position their endosymbiotic partners in the light environment. Previous research on phototaxis in cnidarians has yielded contrasting results. Yamashiro and Nishihara (1995) showed that symbiotic and aposymbiotic Fungiidae corals moved toward light. However, another study with the sea anemone Anthopleura elegantissima found that only symbiotic anemones positioned themselves out of the shade (Pearse 1974). It was unclear whether the host or symbiont was responsible for detecting light and influencing movement of the animal. One clear advantage of using the sea anemone Exaiptasia pallida (Aiptasia) model system for studies on photo-movement is the ability of the aposymbiotic animal to thrive in the absence of its endogenous endosymbiont, which will help establish the origin and characteristics of the light cues and signaling pathways.

Currently, there is a gap in our understanding of the impact of light quality on the position and orientation of cnidarian holobionts in aquatic habitats. Determination of the specific effects of blue and red light is important for our understanding of dinoflagellate rhythmicity and the integration of environmental cues with the physiology of corals living in shallow and deepwater habitats, where underwater light and specific spectral regions decrease exponentially with depth (Sorek and Levy 2012). We use the sea anemone Aiptasia as a model cnidarian-dinoflagellate symbiotic system (Weis et al. 2008; Tolleter et al. 2013;
Baumgarten et al. 2015) to characterize light-stimulated movement of both aposymbiotic animals and holobionts (anemone with endogenous algae plus associated bacteria). Aiptasia harbors and becomes populated with endosymbiotic dinoflagellates that are similar to those that populate reef-building corals (Rowan and Powers 1992; Weis et al. 2008). The objectives of our analyses were to determine the behavioral responses of the anemone under various illumination conditions, establish whether lights of different wavelengths affect holobiont photo-responses, and determine whether these responses are dependent on the presence and type of symbiont harbored by the host. We address the specific hypotheses: (1) Phototropism and phototaxis are dependent on establishment of a holobiont, with no response of aposymbiotic individuals; (2) These photo-responses are affected by light wavelengths and symbiont types.

\section{Materials and methods}

\section{Organisms}

Two Aiptasia types, $\mathrm{H} 2$ and $\mathrm{CC} 7$, were used with various anemone-symbiont combinations: (1) $\mathrm{H} 2$ with its native endosymbiont Breviolum minutum (ITS2 type B1) strain SSB01 (designated H2-SSB01), (2) CC7 with its native endosymbiont Symbiodinium A4 (ITS2 type A4) strain SSA01 (designated CC7-SSA01), (3) CC7 with the heterologous symbiont $B$. minutum strain SSB01 (designated CC7-SSB01), (4) aposymbiotic H2 anemones (designated H2-APO), (5) aposymbiotic CC7 anemones (designated CC7-APO). H2-SSB01 is a clonal, female population derived from a single animal collected in Hawaii (Xiang et al. 2013; Wolfowicz et al. 2016). The other type of Aiptasia, CC7, was male, and collected in Florida. CC7-SSB01 was generated in the laboratory by infecting aposymbiotic CC7 anemones with cultured, axenic B. minutum, which has yielded long-term, stable holobionts (Sunagawa, et al. 2009; Xiang et al. 2013; Wolfowicz et al. 2016). The phylogenetic relationship between these two organisms is unclear, although they were isolated from different geographical locations. Aposymbiotic $\mathrm{H} 2$ and $\mathrm{CC} 7$ anemones were examined by fluorescence microscopy (Leica MZ16FA microscope with Leica EL6000 light source) every week and before commencement of each experiment to confirm the absence of endosymbiotic algae.

Anemones were maintained at a density of 20 to 30 animals in translucent polycarbonate tanks $(10 \times 16 \times 26 \mathrm{~cm}, \quad H \times W \times L, \# 44 \mathrm{CW}$; Cambro, Huntington Beach, USA). The tanks were filled $(2500 \mathrm{~mL})$ with artificial seawater (ASW) (Coral Pro Salt; Red Sea 
Aquatics Ltd, Houston, USA) at 31 to $34 \mathrm{ppt}$ salinity. The organisms were maintained at $26{ }^{\circ} \mathrm{C}$ on a diel $12-\mathrm{h}$ light:12-h dark cycle under white fluorescent bulbs at an intensity of 20 to $25 \mu \mathrm{mol}$ photons $\mathrm{m}^{-2} \mathrm{~s}^{-1}$ of photosynthetically active radiation (PAR), measured with an Apogee PAR quantum meter (MQ-200; Apogee, Logan, USA). With illumination from above, anemones showed no distinctive photo-movement and remained evenly distributed among tanks. Before each experiment, anemones were fed twice a week with freshly hatched Artemia nauplii with water changes within $24 \mathrm{~h}$ of each feeding and complete tank exchange every week. Anemones used for the experiments were grown to a similar size, $0.5 \mathrm{~cm}$ body column diameter. Anemones were not fed during the experimental period due to possible confounding effects of chemical and light cues. Only specific tanks housed the same anemone strain with symbiont-genus associations confirmed through isolation and characterization of DNA (PCR and sequencing) (Xiang et al. 2013).

\section{Phototropism and phototaxis experiments}

Anemones were transferred to black polycarbonate tanks $(10 \times 16 \times 26 \mathrm{~cm}, \quad H \times W \times L, \quad \# 44 \mathrm{CW}$; Cambro, Huntington Beach, USA) filled with 1000-mL ASW and a LED strip light source (Super Bright LEDs Inc.) taped to one end of the tank (Supplementary Figure 1), which created a gradient of light intensity from the front (where the LED strip was placed) to the back of the tank. Light intensities were measured with a light meter (MQ-200; Apogee, Logan, USA). White (Part Number: NFLS-X3LC2-DI-natural white), blue (Part Number: NFLS-X3LC2-DI-blue) and red (Part Number: NFLS-X3-LC2-DIred) LED strips (Super Bright LEDS Inc.) with spectra measured with a radiometer (LI-COR Terrestrial Radiation Sensor with a LI-210 Photometric Sensor) were the sources of illumination. The spectral output for the white LED had two peaks, one in blue at $450 \mathrm{~nm}$ with full width half maximum (FWHM) of $45 \mathrm{~nm}$ and the second in the red at $650 \mathrm{~nm}$ with a FWHM of $175 \mathrm{~nm}$. The peak for the blue LED was $470 \mathrm{~nm}$, FWHM of $62 \mathrm{~nm}$, and the peak for the red LED was $626 \mathrm{~nm}$, FWHM of $36 \mathrm{~nm}$, although there might be low output in the near far red $(680-700 \mathrm{~nm})$, which might impact the phototactic response. For each light color, anemones were placed along a line at equal distances from each other at the position in the tank that received $2 \mu \mathrm{mol}$ photons $\mathrm{m}^{-2} \mathrm{~s}^{-1}$, which allowed for movement both toward and away from the light source. The light intensity used was chosen because it stimulated directional movement in the experimental tanks (pilot experiments) and was within the sensitivity threshold for cnidarian photoperception (Gorbunov and Falkowski 2002; Bessell-Browne et al. 2017). Several photoreceptors encoded on the Symbiodiniaceae genome may be sensing light quality in the holobiont (Xiang et al. 2015).

Anemones were continuously exposed to the light gradient over $11 \mathrm{~d}$, and the tanks were covered with lids to prevent evaporation. The first measurements of anemone positions were taken after $24 \mathrm{~h}$ of exposure to each light treatment. Anemones in each tank were photographed daily, as near to the same time as practicable to allow tracking of individuals over time. Additionally, the number of anemones that bent toward the light was scored daily after $24 \mathrm{~h}$ of exposure to the light treatments. If more than $50 \%$ of the anemone's tentacles and body length was observed to be bent and within the $180^{\circ}$ (first and second quadrants) facing the light, it was considered "bent toward the light." At the conclusion of the experiment, the change in distance $(\mathrm{mm})$ on the $y$ axis between the start and end points for each anemone was quantified using Image $\mathbf{J}$ (Supplementary Figure 1).

As the aim of the experiment was to determine the effect of the symbiont on the host, the control treatment was the response of aposymbiotic anemones to the same experimental conditions. For each replicate trial of anemone strain and light color, eight anemones were placed in the experimental tank and were only housed with anemones of the same type; the total number per anemone type and light color used (including all replicates) are listed in the figures. All experiments were performed in a temperature-controlled growth chamber (HiPoint) at $25{ }^{\circ} \mathrm{C}$, and the salinity and depth of the ASW in the tank were monitored daily. The experiments were repeated over five trials, randomizing the light color used, but always accompanied by the appropriate control; symbiotic anemones were monitored along with control aposymbiotic anemones of the same type under identical light conditions, but in separate tanks. We also attempted to maintain dark control tanks in which anemones were placed in the same positions in the tank as in the equivalent light trial positions. Lids were placed on tanks to exclude the light. After $24 \mathrm{~h}$ in the dark, however, the majority of the symbiotic anemones had detached from the surface of the tank and appeared to be stressed. Thus, dark controls were not meaningful (and not used).

\section{Data analysis}

All statistical analyses were performed using R for Windows (version 3.4.3). For all sets of data, normality was tested with a Shapiro-Wilk test, with residuals plotted and visually inspected. Homogeneity of variance was checked with Levene's test. The assumptions of analysis of variance (ANOVA) were not met as these tests were significant, and thus the nonparametric Kruskal-Wallis test was used. This test relies on the rank ordering of data rather than means and variances; thus, plots show the median and standard 
error of the median determined through bootstrapping data derived from 10,000 trials.

Given the possibility that the algal strains differ in their absorption characteristics in the holobiont, the various anemone-symbiont combinations may not have received an equal intensity of light across the different LEDs, and thus, each anemone-algal association was analyzed separately in each light color. For phototropism experiments, to determine the effect of the symbiont on bending behavior of the anemone, the proportion of anemones that were bent toward the light per day was analyzed with a binomial regression comparing symbiotic type (H2-SSA01, CC7SSA01, CC7-SSB01) versus aposymbiotic (H2-APO, CC7APO) individuals with respect to each light color.

For phototaxis experiments, the distance between the start and end points in the $y$ dimension (i.e., toward or away from the side of the tank with the light; Supplementary Figure 1) for individual anemones was calculated to determine if the directional light caused biased movement. Distances were analyzed with Kruskal-Wallis rank tests separately for each light color, with the anemone type used as the single, fixed factor. Conover pairwise post hoc tests were used to distinguish performances among the different anemone types. Where phototaxis was significant, the probability that the movement toward light significantly deviated from expected outcomes (i.e., 50\%) was compared for symbiotic and aposymbiotic types using a Chisquare analysis.

\section{Results and discussion}

\section{Phototropism under white, blue and red light}

A significantly higher proportion of symbiotic relative to aposymbiotic anemones were bent toward white and blue light after day 1 of the experiment ( $p=0.007, p=0.017$; Fig. 1a, b; Supplementary Figure 2, Supplementary Table 1). Under white light, there was bending of a large proportion of the population and the bending was sustained in the symbiotic organisms over the 11-d treatment period. In blue light, there was a slightly more gradual increase in the proportion of anemones facing the light, although the population of organisms that were bent toward the light also attained near 90\% (for CC7-SSB01 and CC7-SSA01) by the end of the 11-d treatment period. Furthermore, bending toward white and blue light was sustained and more extensive for symbiotic than for aposymbiotic anemones (for the latter, the bending appeared to be directionally random). In contrast, under red light, the proportion of symbiotic and aposymbiotic anemones that were bent toward the light was similar ( $p=0.514$; Fig. 1c, Supplementary Table 1) with the majority of the anemones
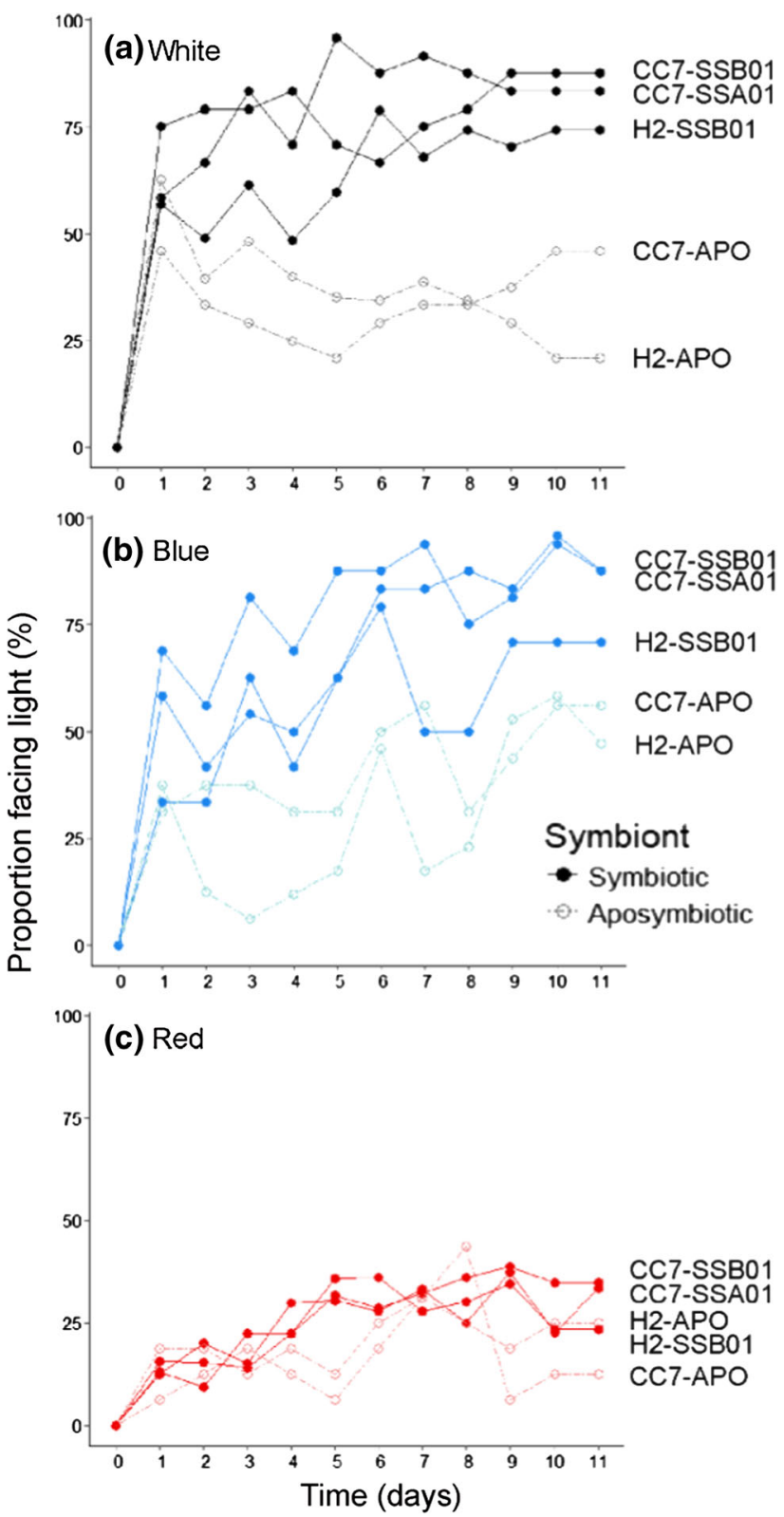

Fig. 1 Proportion of anemones bent toward the light on each day of the experiment. The plots show the proportion of anemones for the aposymbiotic (empty symbols) and the different symbiont associations (filled symbols) that bent toward white (a), blue (b) and red (c) light. See Figs. 2 and 3 for the number of anemones examined for each light color and anemone strain

either bent away from the light or facing directly upwards. These results suggest that sustained phototropism in white and blue light is dependent on the presence of an endosymbiont.

\section{Phototaxis under white light}

In unidirectional white light, the distances that the symbiotic and aposymbiotic anemones moved toward the light were significantly different $(H=21.118, \quad p=0.0003$; 


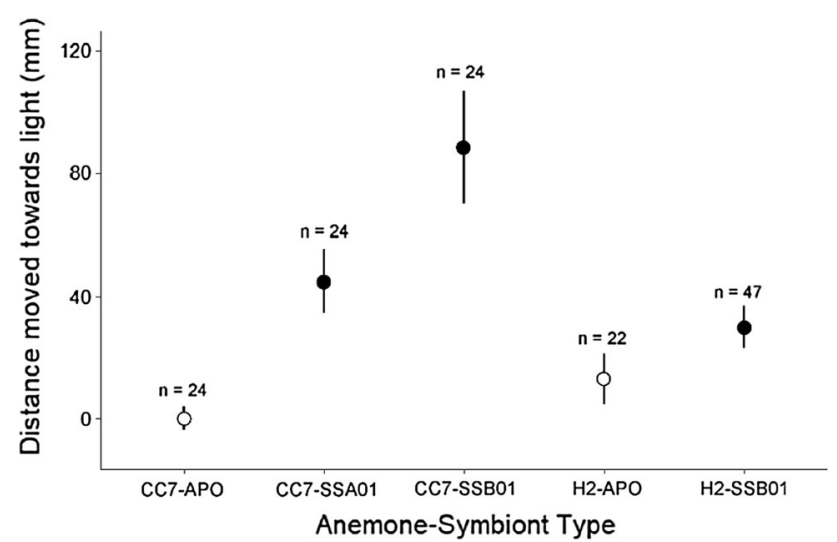

Fig. 2 Phototactic movement of symbiotic (filled symbols) and aposymbiotic (empty symbols) anemones in unidirectional white light. The plot shows the median distance travelled in $\mathrm{mm} \pm \mathrm{SE}$ in the direction of the light for each anemone-symbiont combination

Fig. 2). Comparing the distances moved between different anemone types showed that both CC7-SSA01 and CC7SSB01 moved significantly more toward the light than CC7-APO ( $p=0.034, p=0.000025$, respectively) and H2APO ( $p=0.0326, p=0.0006$, respectively). H2-SSB01 also exhibited greater movement toward the light than $\mathrm{H} 2$ APO, although this difference was not significant ( $p=0.0682$ ) (Fig. 2). CC7-SSB01 moved a significantly different distance than H2-SSB01 ( $p=0.0268)$, indicating that phototaxis of the holobiont appeared to be dependent on both the anemone and symbiont types.

A significantly higher proportion $(82 \%)$ of symbiotic than aposymbiotic anemones moved toward white light, indicating that white light not only influenced the distance moved but also the direction of movement. The proportion of aposymbiotic anemones that exhibited directed movement toward white light did not differ significantly from
$50 \%$, even though aposymbiotic anemones were observed to move large distances. These results suggest that the direction of movement for the aposymbiotic anemones was random. Since the animals only display phototaxis when populated with the algae, the response of the holobiont to light is likely driven by the symbiont.

\section{Phototaxis under blue light}

In unidirectional blue light, the various anemone types moved significantly differently from each other ( $H=11.081, p=0.02566$; Fig. 3), but did not move as far as that observed in white light. Pairwise post hoc tests showed that the influence of B. minutum strain SSB01 on anemone behavior was different depending on the holobiont, where CC7-SSB01 moved closer to the blue light than H2-SSB01 ( $p=0.0117$; Fig. 3). On the other hand, H2-SSB01 moved somewhat less in comparison with the respective aposymbiotic strain (H2-APO, $p=0.0047$; Fig. 3).

In blue light, the response for the two anemone types differed, where B. minutum strain SSB01 performed differently depending on whether it was associated with the $\mathrm{CC} 7$ or $\mathrm{H} 2$ host. When paired with the heterologous host $\mathrm{CC} 7$, there was somewhat greater phototaxis toward blue light (although the response was weak), similar to the response that was observed in white light (which was stronger). The stronger response in white light might be a consequence of the requirement for multiple photoreceptor types to induce a strong phototactic response (Häder et al. 1988; $\mathrm{Ng}$ et al. 2003). When B. minutum strain SSB01 was paired with its natural host $\mathrm{H} 2$, there was little directed movement of either the holobiont or aposymbiotic animals toward blue light. This could be explained if the system is

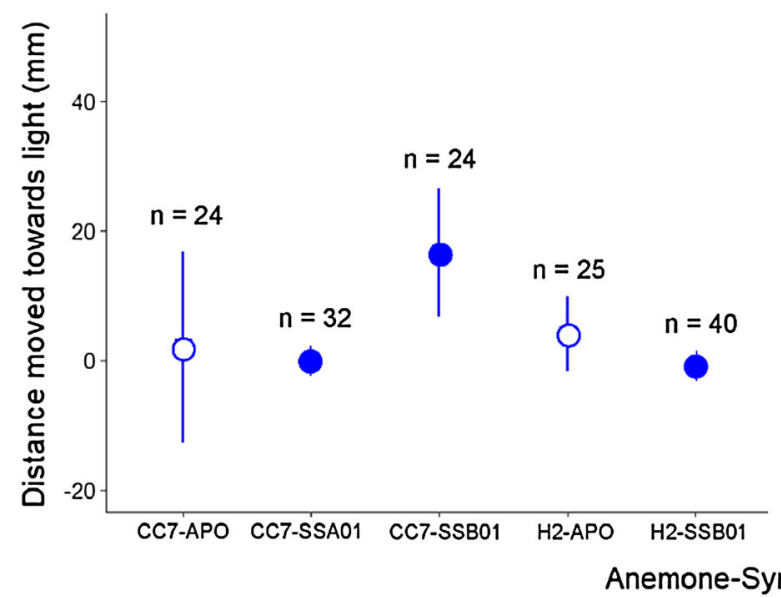

Fig. 3 Phototactic movement of symbiotic (filled symbols) and aposymbiotic (empty symbols) anemones in unidirectional light of different wavelengths. The plot shows the median distance travelled

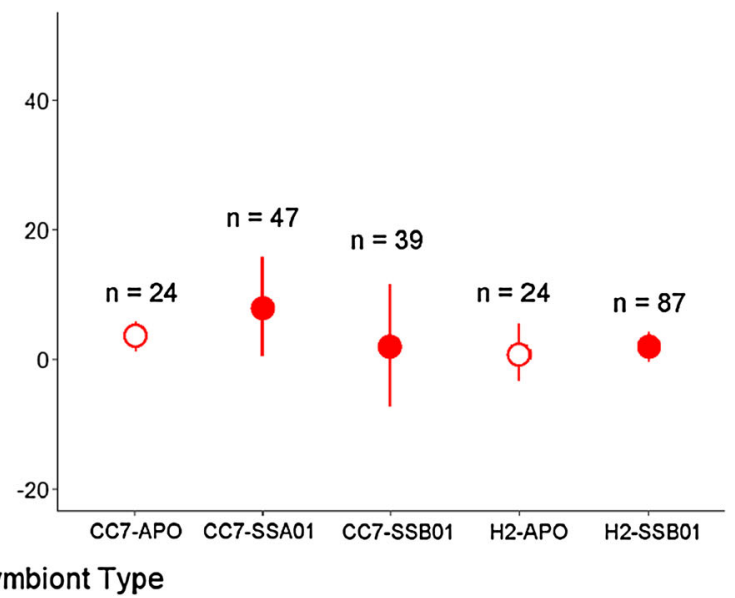

toward the light in $\mathrm{mm} \pm \mathrm{SE}$ of the median for blue (blue points) and red (red points) light for each anemone-symbiont combination 
responding to the need for increased light intensity to maximize photosynthetic efficiency for the heterologous symbiont (but not for the homologous symbiont; SSB01 with $\mathrm{H} 2$ and SSA01 with CC7), although even the holobiont with homologous symbionts did show movement toward white light (Fig. 2). Detailed analysis of photosynthetic efficiencies at different light intensities and qualities would be required to examine this issue.

\section{Phototaxis under red light}

In unidirectional red light, none of the anemone types performed significantly different from any of the others ( $H=0.6026, p=0.9628$; Fig. 3$)$; there was no directional phototaxis displayed. Hence, a red light photoreceptor might not be involved in the control of phototaxis (although it is possible that movement is impacted by a low level of output from the LED in the 680-700-nm region of the spectrum). Also, phototaxis can involve multiple photoreceptors and considerable amplification in its sensory transduction chain (Häder 1986; Bhaya 2004), which would make white light more effective than individual narrow spectrum light qualities (e.g., blue or red). It is also possible that higher intensity blue or red light would stimulate stronger phototactic movement (Sineschchekov et al. 2002). Finally, the action spectrum of the algae (lightdriven photosynthesis) is mostly due to chlorophylls, which absorb in the red and blue regions of the spectrum, and the xanthophylls, which absorb at $\sim 470 \mathrm{~nm}$ (Venn, et al. 2006; Wangpraseurt et al. 2014). Thus, although anemones were placed at the position receiving $2-\mu \mathrm{mol}$ photons $\mathrm{m}^{-2} \mathrm{~s}^{-1}$, this may have not been equivalent across the different colored light sources due to the absorption characteristics of the two different algae. However, the pigmentation of the two algal types is very similar, and therefore, the different responses to light among the anemone-symbiont combinations are more likely a consequence of different interactions between the animal and the symbiont.

Both SSA01 and SSB01 possess putative rhodopsins, phytochrome-like and cryptochrome proteins, although other or unusual photoreceptors might also be responsible for sensing the light signal (Duanmu et al. 2017). There are a higher number of putative cryptochrome encoding genes in SSA01 and SSB01 (20 in A4: Xiang, T. unpublished data; 18 in B1: Xiang, et al. 2015) than genes encoding other identified photoreceptors, which could reflect an increased number of algal responses to blue light. Furthermore, the importance of cryptochrome photoreceptors in dinoflagellates is suggested by the finding that changes in blue light controlled photosynthetic rhythms in the Symbiodiniaceae correspond to changes in cryptochrome gene expression (Sorek and Levy 2012).

\section{Phototaxis in Aiptasia, the role of the algae and its impact in nature}

In white light, only symbiotic anemones displayed phototaxis and phototropism. The lack of bending or movement of aposymbiotic anemones toward the light suggests the loss of a signal responsible for the movement. As anemones lack specialized, visual structures, it is uncertain if they can perceive the direction of light, although they do harbor opsins, a group of light-sensitive proteins important for vision in other organisms (Baumgarten et al. 2015). Given our results, it seems more likely that the algae are responsible for the light perception that drives movement of the animal. In addition to having multiple photoreceptors, as mentioned above, crystalline deposits of uric acid in the Symbiodiniaceae may function as eyespots that perceive the light vector (Yamashita et al. 2009).

The algae also translocate glucose and other metabolites (Burriesci et al. 2012), which could influence phototaxis and phototropism of the anemone; in turn, the ability to move and orient toward the light could stimulate photosynthesis and continued production and translocation of sugars from the algae to the host. The rapid transfer of synthesized glucose from the symbiont to the anemone is different between anemone-symbiont combinations (Burriesci et al. 2012), which may reflect differences in the integration of the metabolisms of the specific symbionthost partners (Matthews et al. 2018). These findings could help explain differences in the light responses of $\mathrm{CC} 7$ and $\mathrm{H} 2$ anemone types.

For anemones, phototaxis in the field could allow for significant relocation and spreading, which is congruent with our findings that anemones can move whole tank distances $(226 \mathrm{~mm})$ within $24 \mathrm{~h}$. The slow, sliding pedal disk movement displayed by Aiptasia would also likely remain steady in turbulent environments. Advantages associated with phototactic behavior would involve escape from shaded cavities and selection of favorable habitats and light conditions for growth and reproduction. Additionally, when symbiotic anemones are placed in the shade or dark, the number of algae within the host tissue and total chlorophyll continually decrease (Buchsbaum 1968). Thus, the ability to phototax would allow sustained maintenance of a robust symbiotic association and the production of fixed carbon.

Differential responses of different host and symbiont types to light would presumably impact the overall distributions of these organisms on both local and larger scales and be important for niche habitation for anemones, as well as for scleractinian coral planulae, which can show lightdependent settlement (Mundy and Babcock 1998). Therefore, while phototaxis may not be a relevant trait for adult scleractinian corals, the differential responses of host types 
and the effect of symbiont type on animal behavior could affect the overall distributions of these cnidarians on a global scale.

\section{Conclusions}

Our results show that aspects of phototropism and phototaxis in the sea anemone Aiptasia under white and blue light are dependent on a functional symbiosis, with the symbiont likely influencing a sustained, directed behavioral response of the anemone to light. The effect of the symbiont on the phototropic and phototactic responses differed depending on the host; thus it cannot be assumed that the same phenotype will be elicited when different symbionts are paired with various host animals. As enhancing the genome of coral symbionts and assisted evolution have been suggested as potential ways to increase reef resilience in the face of climate change (van Oppen et al. 2015; Levin et al. 2017), it will be critical to better understand hostsymbiont interactions. Aiptasia provides a strong model system to explore these interactions.

The sensing of light quality may be associated with specific photoreceptors, synthesized by the symbiont, that elicit signaling resulting in directional photo-movement, a response that does not occur in aposymbiotic animals. Other factors that might impact the light responses are the intensity of the illumination and the synthesis and translocation of photosynthetically-derived molecules. The ability to perform phototropism and phototaxis provides the anemones with the capacity to seek out favorable habitats, allowing the holobiont to better exploit environmental light conditions; this process does appear to depend on the type of anemone, the genus/strain of the symbiont and the wavelength of light. Overall, these results enhance our understanding of the influence of photosynthetic symbionts on the direction and amount of movement of its animal partner in response to light signals.

\begin{abstract}
Acknowledgements We thank Tingting Xiang for Symbiodinium clade A sequences and members of the Pringle Lab at Stanford University for helpful discussion and culture of anemones. This study was supported by a Carnegie Venture Grant and an award from the Gordon and Betty Moore Foundation, Grant No. 2629.01.
\end{abstract}

Open Access This article is distributed under the terms of the Creative Commons Attribution 4.0 International License (http://crea tivecommons.org/licenses/by/4.0/), which permits unrestricted use, distribution, and reproduction in any medium, provided you give appropriate credit to the original author(s) and the source, provide a link to the Creative Commons license, and indicate if changes were made.

\section{References}

Anthony K, Connolly SR, Hoegh-Guldberg O (2007) Bleaching, energetics, and coral mortality risk: effects of temperature, light, and sediment regime. Limnol Oceanogr 52:716-726

Baumgarten S, Simakov O, Esherick LY, Liew YJ, Lehnert EM, Michell CT, Li Y, Hambleton EA, Guse A, Oates ME, Gough J, Weis VM, Aranda M, Pringle JR, Voolstra CR (2015) The genome of Aiptasia, a sea anemone model for coral symbiosis. PNAS 112:11893-11898

Bessell-Browne P, Negri AP, Fisher R, Clode PL, Jones R (2017) Impacts of light limitation on corals and crustose coralline algae. Scientific Reports 7:11553

Bhaya D (2004) Light matters: phototaxis and signal transduction in the unicellular cyanobacteria. Molecular Microbiology 53:745-754

Brown BE (1997) Coral bleaching: causes and consequences. Coral Reefs 16:129-138

Buchsbaum VM (1968) Behavioural and physiological responses to light by the sea anemone Anthopleura elegantissima as related to its algal endosymbionts. Ph.D. Diss. Stanford University, Stanford

Burriesci MS, Raab TK, Pringle JR (2012) Evidence that glucose is the major transferred metabolite in dinoflagellate-cnidarian symbiosis. Journal of Experimental Biology 215:3467-3477

Christie JM, Blackwood L, Petersen J, Sullivan S (2015) Plant flavoprotein photoreceptors. Plant Cell Physiol 56:401-413

Cohen I, Dubinsky Z, Erez J (2016) Light enhanced calcification in hermatypic corals: new insights from light spectral responses. Front Mar Sci 2:122

Delvin P, Kay S (2000) Cryptocromes are required for phytochrome signaling to the circadian clock but not for rhythmicity. Plant Cell 12:2499-2509

Duanmu D, Rockwell NC, Lagarias JC (2017) Algal light sensing and photoacclimation in aquatic environments. Plant Cell Environment 40:2558-2570

Gattuso J-P, Pichon M, Frankignoulle M (1995) Biological control of air-sea fluxes: effects of photosynthetic and calcifying marine organisms and ecosystems. Mar Ecol Prog Ser 129:307-312

Gorbunov MY, Falkowski PG (2002) Photoreceptors in the cnidarian hosts allow symbiotic corals to sense blue moonlight. Limnol Oceanogr 47:309-315

Häder DP, Watanabe M, Furuya M (1988) Multiple Photoreceptors in Phototaxis of Dictyostelium discoideum Amoebae. In: Tazawa M (ed) Cell Dynamics. Protoplasma, vol 1. Springer, Vienna

Häder D-P (1986) Signal perception and amplification in photomovement of prokaryotes. Biochimica et Biophysica Acta (BBA) Reviews on Biomembranes 864:107-122

Hastings J, Sweeny B (1960) The action spectrum for shifting the phase of the rhythm of luminescence in Gonyaulax polyedra. J Gen Physiol 43:697-706

Hoegh-Guldberg O, Mumby PJ, Hooten AJ, Steneck RS, Greenfield P, Gomez E, Harvell CD, Sale PF, Edwards AJ, Caldeira K, Knowlton N, Eakin CM, Iglesias-Prieto R, Muthiga N, Bradbury RH, Dubi A, Hatziolos ME (2007) Coral reefs under rapid climate change and ocean acidification. Science 318:1737-1742

Iglesias-Prieto R, Beltran VH, LaJeunesse TC, Reyes-Bonilla H, Thome PE (2004) Different algal symbionts explain the vertical distribution of dominant reef corals in the eastern Pacific. Proceedings of the Biological Society 271:1757-1763

Kong SG, Okajima K (2016) Diverse photoreceptors and light responses in plants. J Plant Res 129:111-114

Levin RA, Voolstra CR, Agrawal S, Steinberg PD, Suggett DJ, van Opeen MJH (2017) Engineering Strategies to Decode and 
Enhance the Genomes of Coral Symbionts. Frontiers in Microbiology 8:1220

López-Figueroa F, Conde-Álvarez R, Gómez I (2003) Relations between electron transport rates determined by pulse amplitude modulated chlorophyll fluorescence and oxygen evolution in macroalgae under different light conditions. Photosynthesis Research 75:259-275

Matthews JL, Oakley CA, Lutz A, Hillyer KE, Roessner U, Grossman AR, Weis VM, Davy SK (2018) Partner switching and metabolic flux in a model cnidarian-dinoflagellate symbiosis. Proc Biol Sci 28:20182336

Mundy CN, Babcock RC (1998) Role of light intensity and spectral quality in coral settlement: Implications for depth-dependent settlement? Journal of Experimental Marine Biology and Ecology 223:235-255

Ng WO, Grossman AR, Bhaya D (2003) Multiple light inputs control phototaxis in Synechocystis sp. strain PCC6803. J Bacteriol 185:1599-1607

Pearse VB (1974) Modification of sea anemone behaviour by symbiotic zooxanthellae: phototaxis. Biol Bull 147:630-640

Roth MS (2014) The engine of the reef: photobiology of the coralalgal symbiosis. Front Microbiol 5:422

Rowan R, Powers D (1992) Ribosomal RNA sequences and the diversity of symbiotic dinoflagellates (zooxanthellae). Proc Natl Acad Sci 89:3639-3643

Sawyer SJ, Dowse HB, Shick M (1994) Neurophysiological correlates of the behavioural response to light in the sea anemone Anthopleura elegantissima. Biol Bull (Woods Hole, Mass) 186:195-201

Shick JM (1991) A functional biology of sea anemones. Chapman \& Hall, London

Sineschchekov OA, Jung K-H, Spudich JL (2002) Two rhodopsins mediate phototaxis to low- and high-intensity light in Chlamydomonas reinhardtii. Proc Natl Acad Sci USA 99:8689-8694

Sorek M, Levy O (2012) Influence of the Quantity and Quality of Light on Photosynthetic Periodicity in Coral Endosymbiotic Algae. PLoS ONE 7:e43264

Somers D, Delvin P, Kay S (1998) Phytochromes and cryptochromes in the entrainment of the Arabidopsis circadian clock. Science 282:1488-1490

Su N, Wu Q, Shen Z, Xia K, Cui J (2014) Effects of light quality on the chloroplastic ultrastructure and photosynthetic characteristics of cucumber seedlings. Plant Growth Regulation 73:227-235

Sunagawa S, Wilson EC, Thaler M, Smith ML, Caruso C, Pringle JR, Weis VM, Medina M, Schwarz JA (2009) Generation and analysis of transcriptomic resources for a model system on the rise: the sea anemone Aiptasia pallida and its dinoflagellate endosymbiont. BMC Genomics 10:258
Tolleter D, Seneca FO, DeNofrio JC, Krediet CJ, Palumbi SR, Pringle JR, Grossman AR (2013) Coral Bleaching Independent of Photosynthetic Activity. Current Biology 23:1782-1786

van Oppen MJH, Oliver JJ, Putnam HM, Gates RD (2015) Building coral reef resilience through assisted evolution. PNAS 112:2307-2313

Venn AA, Wilson MA, Trapido-Rosenthal HG, Keely BJ, Douglas AE (2006) The impact of coral bleaching on the pigment profile of the symbiotic alga, Symbiodinium. Plant, Cell and Environment 29:2133-2142

Wangpraseurt D, Tamburic B, Szabo M, Suggett D, Ralph PJ, Kuhl M (2014) Spectral effects on Symbiodinium photobiology studied with a programmable light engine. PLoS ONE 9:e112809

Warner ME, Suggett DJ (2016) The Photobiology of Symbiodinium spp.: linking physiological diversity to the implications of stress and resilience. In: Goffredo S, Dubinsky Z (eds) The Cnidaria, Past, Present and Future. Springer, Cham, pp 489-509

Weis VM, Davy SK, Hoegh-Guldberg O, Rodriguez-Lanetty M, Pringle JR (2008) Cell biology in model systems as the key to understanding corals. Trends Ecol Evol 23:369-376

Wijgerde T, van Melis A, Silva CIF, Leal MC, Vogels L, Mutter C, Osinga R (2014) Red light represses the photophysiology of the scleractinian coral Stylophora pistillata. PLoS ONE 9:e92781

Wolfowicz I, Baumgarten S, Voss PA, Hambleton EA, Voolstra CR, Hatta M, Guse A (2016) Aiptasia sp. larvae as a model to reveal mechanisms of symbiont selection in cnidarians. Scientific Reports 6:32366

Xiang T, Hambleton EA, DeNofrio JC, Pringle JR, Grossman AR (2013) Isolation of clonal, axenic strains of the symbiotic dinoflagellate Symbiodinium and their growth and host specificity. J Phycol 49:447-458

Xiang T, Nelson W, Rodriguez J, Tolleter D, Grossman AR (2015) Symbiodinium transcriptome and global responses of cells to immediate changes in light intensity when grown under autotrophic or mixotrophic conditions. Plant J 82:67-80

Yacobovitch T, Benayahu Y, Weis VM (2004) Motility of zooxanthellae isolated from the Red Sea soft coral Heteroxenia fuscescens (Cnidaria). J Exp Mar Biol Ecol 298:35-48

Yamashiro H, Nishihara M (1995) Phototaxis in Fungiidae corals (Scleractinia). Mar Biol 124:461-464

Yamashita H, Kobiyama A, Koike K (2009) Do uric acid deposits in zooxanthellae function as eye-spots? PLoS ONE 4:e6303

Publisher's Note Springer Nature remains neutral with regard to jurisdictional claims in published maps and institutional affiliations. 